\title{
A Novel Linear Algebra Method for the Determination of Periodic Steady States of Nonlinear Oscillators
}

\begin{abstract}
Periodic steady-state (PSS) analysis of nonlinear oscillators has always been a challenging task in circuit simulation. We present a new way that uses numerical linear algebra to identify the PSS(s) of nonlinear circuits. The method works for both autonomous and excited systems. Using the harmonic balancing method, the solution of a nonlinear circuit can be represented by a system of multivariate polynomials. Then, a Macaulay matrix based root-finder is used to compute the Fourier series coefficients. The method avoids the difficult initial guess problem of existing numerical approaches. Numerical examples show the accuracy and feasibility over existing methods.
\end{abstract}

Keywords-Steady-state analysis, autonomous oscillator, nonlinear circuit simulation, Macaulay matrix

\section{INTRODUCTION}

Accurate simulation of the behaviors of microwave and radio-frequency $(\mathrm{RF})$ circuits has become a key issue in mixedsignal circuit design due to the exponential growth of the complexity in the past few years. Periodic steady-state (PSS) analysis plays a critical role, especially in the simulation of RF communication circuits [1].

Two specialized PSS algorithms, namely, shooting Newton (SN) and harmonic balance (HB) [2]-[5] are deployed in traditional SPICE simulators for decades. They are well developed for the PSS analysis of excited systems, i.e., systems with certain periodic inputs. In such systems, the period of the PSS is always forced to be the same as that of the input. SN will then iteratively shoot for aligning the initial and final values over the given period, while HB numerically solves for the Fourier series coefficients with respect to the known period. However, it is difficult for traditional SN and HB to work under an unknown oscillating frequency. Therefore, they cannot readily be used to determine the PSS of autonomous systems (i.e., systems without external inputs) such as oscillators in RF communication circuits.

Several modifications have been made for $\mathrm{SN}$ and $\mathrm{HB}$ to adapt to autonomous systems by treating the unknown oscillation frequency as a new state variable [6]-[9]. These methods still require solving systems of nonlinear equations via the Newton-Raphson method, which highly depends on the initial guess. There might be multiple solutions to the nonlinear equations where only one is stable. Therefore it is always hard to choose an initial value of the frequency in the right basin of attraction, especially for RF oscillators.

To avoid dependence on the initial guess when solving nonlinear systems numerically, other methods, such as Volterra series [10], [11], have been proposed. The oscillation frequency is then obtained by solving a univariate equation which consists of Volterra transfer functions. However, Volterra series approximation might be inaccurate, especially for highly nonlinear oscillators. Moreover, Volterra transfer functions suffer from singularity problems when applied to descriptor systems. At the same time, algebraic geometry has been used to solve polynomial systems without any initial guesses. For example in [12], a polynomial system of harmonic balancing equations is solved using a Groebner basis. Nonetheless, although no initial guesses are needed in the method, Groebner bases can only deal with integer coefficients. In addition, Groebner bases typically generate polynomials with very large coefficients (usually hundreds of digits), which is generally inconvenient to use in engineering.

On the other hand, a new numerical way of finding all the roots of a multivariate polynomial system has been recently introduced in [13], [14]. This method uses only numerical linear algebra and works with arbitrary real coefficients while finding all the roots by computing the null space of the Macaulay matrix. The main computational tools in this method are either the singular value decomposition (SVD) or the rankrevealing QR decomposition, for which numerically backward stable implementations exist [15].

In this paper, a novel linear algebraic method is proposed for the PSS analysis of nonlinear oscillators. First, a general nonlinear system is transformed into an equivalent polynomial differential algebraic equation (DAE) system. Then, a polynomial system is constructed from the DAE system via harmonic balancing equations. After that, the Macaulay matrix based root-finder is used to solve for the Fourier series coefficients from the polynomial system. The proposed method completely avoids initial guesses as in iterative numerical solvers such as $\mathrm{SN}$ and $\mathrm{HB}$, and works perfectly with arbitrary system coefficients. Moreover, it is able to find all solutions as long as the PSS of the system exists.

The paper is organized as follows. Section II reviews the background preliminaries of the polynomial DAE system. Then, our numerical linear algebra method is proposed in Section III. Numerical examples are given in Section IV. Several notable remarks are presented in Section V. Section VI concludes this paper.

\section{BACKGROUND AND PRELIMINARIES}

\section{A. Systems of polynomial differential algebraic equations}

First, we start with a general nonlinear DAE system

$$
F(x, \dot{x}, \ddot{x}, \ldots, u)=\mathbf{0},
$$


where $x \in \mathbb{R}^{\tilde{n}}, u \in \mathbb{R}^{l}$ are the state vector and input vector, respectively. $F(\cdot)$ is an arbitrary nonlinear vector function. In practical circuit modeling, $F(\cdot)$ usually satisfies certain common nonlinearities, such as polynomial, rational, sinusoidal and exponential etc. For instance, diodes are usually modeled by an exponential nonlinear function $i_{D}=I_{s}\left(e^{v_{D} /\left(n V_{T}\right)}-1\right)$ and typical MOSFET models include recursive polynomial, rational and exponential nonlinearities. It is proven in [16] that if the DAE system (1) complies such condition, it can always be converted to another equivalent DAE system with only polynomial nonlinearities

$$
F_{p}(x, \dot{x}, \ddot{x}, \ldots, u)=\mathbf{0},
$$

where $x \in \mathbb{R}^{n}, u \in \mathbb{R}^{l}$ and $F_{p}(\cdot)$ is a polynomial vector function of $u, x, \dot{x}, \ddot{x}$, etc.

The best way to illustrate the process is by means of an example. Suppose we have a "diode-type" exponential nonlinear system

$$
F(z, u)=-\dot{z}-z-e^{4 z}+1+u=0,
$$

wherein $u$ is a scalar input and $z$ the scalar state variable. By setting $z_{1}=z, z_{2}=e^{4 z_{1}}-1$ and $x=\left(z_{1}, z_{2}\right)^{T}$ such that $\dot{z}_{2}=4 e^{4 z_{1}} \dot{z}_{1}=4\left(z_{2}+1\right)\left(-z_{1}-z_{2}+u\right)$, we end up with the polynomial DAE system

$$
\begin{aligned}
F_{p}(x, \dot{x}, u) & =C_{0} \dot{x}+G_{1} x+G_{2} x \otimes x+D_{1} x \otimes u+B u=\mathbf{0} \\
\text { with } C_{0} & =\left(\begin{array}{cc}
-1 & 0 \\
0 & -1
\end{array}\right), G_{1}=\left(\begin{array}{cc}
-1 & -1 \\
-4 & -4
\end{array}\right) \\
G_{2} & =\left(\begin{array}{cccc}
0 & 0 & 0 & 0 \\
0 & -4 & 0 & -4
\end{array}\right), D_{1}=\left(\begin{array}{ll}
0 & 0 \\
0 & 4
\end{array}\right), B=\left(\begin{array}{l}
1 \\
4
\end{array}\right),
\end{aligned}
$$

where the $\otimes$ symbol denotes the Kronecker product.

For notational ease, we will illustrate our proposed algorithm on the following simplified $F_{p}(\cdot)^{1}$

$$
\begin{aligned}
F_{p}(x, \dot{x}, \ddot{x}, u)= & C_{0} \dot{x}+C_{1} x \otimes \dot{x}+C_{2} x^{(2)} \otimes \dot{x}+E_{0} \ddot{x}+G_{1} x \\
& +G_{2} x^{(2)}+G_{3} x^{(3)}+D_{1} x \otimes u+D_{2} x^{(2)} \otimes u \\
& +B u=\mathbf{0},
\end{aligned}
$$

where $C_{i} \in \mathbb{R}^{n \times n^{i+1}}, E_{0} \in \mathbb{R}^{n \times n}, G_{i} \in \mathbb{R}^{n \times n^{i}}, D_{i} \in \mathbb{R}^{n \times l n^{i}}$ and $B \in \mathbb{R}^{n \times l}$ are all constant matrices. All derivations in this paper can easily accommodate higher order powers or cross products of $u, x, \dot{x}, \ddot{x}$, etc. A formal proof and procedure on how to convert the original DAE system into an equivalent polynomial system can be found in [16].

\section{LINEAR ALGEBRA PSS FINDER}

\section{A. Harmonic balancing equation}

We are now ready to set up a system of multivariate polynomials from (4) by the harmonic balancing method.

Suppose the PSS solution of $x$ in (4) can be approximated by a truncated Fourier series

$$
x(t)=\sum_{k=-m}^{m} p_{k} e^{j k \omega t},
$$

where $p_{k} \in \mathbb{C}^{n}$ are vectors of complex coefficients and $\omega$ is a constant if the frequency of the input $u$ is known. For

\footnotetext{
${ }^{1}$ We use the shorthand $x \otimes x=x^{(2)}$ etc. throughout the paper.
}

autonomous systems, $\omega$ can be regarded as an extra unknown variable. Since for any real signal $x(t)$, its coefficients in the Fourier bases $e^{j k \omega t}$ and $e^{-j k \omega t}$ must be a pair of complex conjugates, we can specifically define

$$
p_{0}=p_{0 r}, p_{ \pm k}=p_{k r} \pm j p_{k i}, k=1, \ldots, m,
$$

where $p_{0 r}, p_{k r}, p_{k i} \in \mathbb{R}^{n}$. We further define the coefficients matrix $P=\left(p_{-m}, p_{-m+1}, \ldots, p_{0}, \ldots, p_{m-1}, p_{m}\right)$ and the Fourier basis $\Phi_{m}(t)=\left(e^{-j m \omega t}, \ldots, e^{j m \omega t}\right)^{T}$, thus $x(t)=$ $P \Phi_{m}$. Similarly, a periodic input $u$ can always be expressed under the Fourier basis as $u=U \Phi_{m}$.

The differential operational matrix under the Fourier basis is then defined as

$$
D_{m}=j \omega \operatorname{diag}([-m,-m+1, \ldots, m-1, m]) .
$$

It can be found that the time derivative of the basis functions $\Phi_{m}$ is $D_{m} \Phi_{m}$. Therefore $\dot{x}$ and $\ddot{x}$ are simply $P D_{m} \Phi_{m}$ and $P D_{m}^{2} \Phi_{m}$, respectively.

The Kronecker power $x^{(2)}$ can be written as

$$
x^{(2)}=\left(P \Phi_{m}\right)^{(2)}=\left(P \Phi_{m}\right) \otimes\left(P \Phi_{m}\right)=P^{(2)} \Phi_{m}{ }^{(2)} \text {. }
$$

The Kronecker product of the Fourier basis $\Phi_{m}$ (2) can be simplified to a selector matrix, denoted $S_{m, m}$, times $\Phi_{2 m}$. For example the selector matrix $S_{m, m}$ when $m=1$ is

$$
\begin{aligned}
& \Phi_{1}{ }^{2}=\left(\begin{array}{c}
e^{-j \omega t} \\
1 \\
e^{j \omega t}
\end{array}\right) \otimes\left(\begin{array}{c}
e^{-j \omega t} \\
1 \\
e^{j \omega t}
\end{array}\right) \\
& =\left(\begin{array}{lllllllll}
1 & 0 & 0 & 0 & 0 & 0 & 0 & 0 & 0 \\
0 & 1 & 0 & 1 & 0 & 0 & 0 & 0 & 0 \\
0 & 0 & 1 & 0 & 1 & 0 & 1 & 0 & 0 \\
0 & 0 & 0 & 0 & 0 & 1 & 0 & 1 & 0 \\
0 & 0 & 0 & 0 & 0 & 0 & 0 & 0 & 1
\end{array}\right) \quad\left(\begin{array}{c}
e^{-j 2 \omega t} \\
e^{-j \omega t} \\
1 \\
e^{j \omega t} \\
e^{j \omega t}
\end{array}\right)=S_{1,1} \Phi_{2} .
\end{aligned}
$$

Similarly, the Kronecker product of the Fourier basis $\Phi_{m}{ }^{(3)}$ can be simplified to

$\Phi_{m}^{(3)}=S_{m, m} \Phi_{2 m} \otimes \Phi_{m}=S_{m, m} S_{2 m, m} \Phi_{3 m} \triangleq S_{m, m, m} \Phi_{3 m}$.

High order Kronecker products can be constructed in the same way. It should be noticed that instead of growing exponentially, the basis dimension increases only linearly by taking the advantage of selector matrices. Therefore, we have the following equalities

$$
\begin{aligned}
x & =P \Phi_{m}, \\
\dot{x} & =P D_{m} \Phi_{m}, \\
\ddot{x} & =P D_{m}^{2} \Phi_{m}, \\
x^{(2)} & =P^{(2)} S_{m, m} \Phi_{2 m}, \\
x \otimes \dot{x} & =P^{(2)}\left(I_{m} \otimes D_{m}\right) S_{m, m} \Phi_{2 m}, \\
x \otimes u & =(P \otimes U) S_{m, m} \Phi_{2 m}, \\
x^{(3)} & =P^{(3)} S_{m, m, m} \Phi_{3 m}, \\
x^{(2)} \otimes \dot{x} & =P^{(3)}\left(I_{2 m} \otimes D_{m}\right) S_{m, m, m} \Phi_{3 m}, \\
x^{(2)} \otimes u & =\left(P^{(2)} \otimes U\right) S_{m, m, m} \Phi_{3 m},
\end{aligned}
$$


Replacing the corresponding terms in (4), the original equation can be written in the Fourier basis as

$$
\begin{aligned}
\mathbf{0} & =C_{0} P D_{m} \Phi_{m}+C_{1} P^{(2)}\left(I_{m} \otimes D_{m}\right) S_{m, m} \Phi_{2 m} \\
& +C_{2} P^{(3)}\left(I_{2 m} \otimes D_{m}\right) S_{m, m, m} \Phi_{3 m}+E_{0} P D_{m}^{2} \Phi_{m} \\
& +G_{1} P \Phi_{m}+G_{2} P^{(\mathcal{2})} S_{m, m} \Phi_{2 m}+G_{3} P^{(3)} S_{m, m, m} \Phi_{3 m} \\
& +D_{1}(P \otimes U) S_{m, m} \Phi_{2 m}+D_{2}\left(P^{(2)} \otimes U\right) S_{m, m, m} \Phi_{3 m}+B U \Phi_{m} .
\end{aligned}
$$

Since we are interested in the coefficients with respect to the basis $\Phi_{m}$, it is necessary to truncate all other bases with an order higher than $m$ or lower than $-m$. The truncation can be achieved by removing the columns in each $S$ that correspond to elements that are not in the basis $\Phi_{m}$. Suppose the new selector matrix is $\tilde{S}$ after the truncation, (10) then becomes

$$
\begin{aligned}
\mathbf{0} & =C_{0} P D_{m} \Phi_{m}+C_{1} P^{(2)}\left(I_{m} \otimes D_{m}\right) \tilde{S}_{m, m} \Phi_{m} \\
& +C_{2} P^{(3)}\left(I_{2 m} \otimes D_{m}\right) \tilde{S}_{m, m, m} \Phi_{m}+E_{0} P D_{m}^{2} \Phi_{m} \\
& +G_{1} P \Phi_{m}+G_{2} P^{(2)} \tilde{S}_{m, m} \Phi_{m}+G_{3} P^{(3)} \tilde{S}_{m, m, m} \Phi_{m} \\
& +D_{1}(P \otimes U) \tilde{S}_{m, m} \Phi_{m}+D_{2}\left(P^{(2)} \otimes U\right) \tilde{S}_{m, m, m} \Phi_{m}+B U \Phi_{m} .
\end{aligned}
$$

The $\Phi_{m}$ is a common factor to each term in (11) and can hence be removed to arrive at the harmonic balancing equation

$$
\begin{aligned}
\mathbf{0} & =C_{0} P D_{m}+C_{1} P^{(2)}\left(I_{m} \otimes D_{m}\right) \tilde{S}_{m, m} \\
& +C_{2} P^{(3)}\left(I_{2 m} \otimes D_{m}\right) \tilde{S}_{m, m, m}+E_{0} P D_{m}^{2} \\
& +G_{1} P+G_{2} P^{(2)} \tilde{S}_{m, m}+G_{3} P^{(3)} \tilde{S}_{m, m, m} \\
& +D_{1}(P \otimes U) \tilde{S}_{m, m}+D_{2}\left(P^{(2)} \otimes U\right) \tilde{S}_{m, m, m}+B U .
\end{aligned}
$$

It should be noted that both sides of (12) are $n$-by- $(2 m+1)$ matrices. Therefore, after separating the real and imaginary parts of (12), (12) can be regarded as a system of $n(2 m+1)$ multivariate polynomials with $n(2 m+1)$ unknowns $\left(p_{0 r}, p_{k r}\right.$ and $\left.p_{k i}\right)$.

For autonomous cases, the extra variable $\omega$ needs to be introduced, which makes (12) underdetermined. This results in an infinite amount of solutions, which come from two sources. First, for autonomous systems, any arbitrary phase shift of a solution to (12) will also be a valid solution to the original system. Therefore, to enforce the solutions be isolated from one another, it is necessary to fix the phase of the solution by, e.g., setting the first element ${ }^{2}$ of $p_{1 i}$ to 0 (forcing the phase of the first order harmonic of the first state variable to be 0 or $\pi$ ). Note that setting $p_{1 i}$ to 0 in (12) and the addition of $\omega$ results in a polynomial system of $n(2 m+1)$ equations and unknowns. Second, it is readily found that each harmonic order of the fundamental frequency is also a valid oscillating frequency. In other words, if $\left(\omega, p_{1}, p_{2}, p_{3}, p_{4}, \ldots\right)$ is a set of solutions to (12), then so are $\left(2 \omega, 0, p_{1}, 0, p_{2}, 0, p_{3}, \ldots\right)$ and $\left(3 \omega, 0,0, p_{1}, 0,0, p_{2}, \ldots\right)$, etc. Thus, to ensure $\omega$ to be the fundamental oscillating frequency, we set the first element of $p_{1 r}$ nonzero by adding an extra polynomial equation $h \cdot p_{1 r}^{1}-1=0$, where $h$ is an extra variable.

In this way autonomous systems will have $n(2 m+1)+1$ polynomials and unknowns, while forced systems will have $n(2 m+1)$ polynomials and unknowns. For the remainder of this article we denote the number of polynomials and unknowns by $\hat{n}$ and consider systems of $\hat{n}$ polynomials in the variables $x_{1}, \ldots, x_{\hat{n}}$.

\footnotetext{
${ }^{2}$ We denote $p_{1 i}^{1}$ to be the first element of the vector $p_{1 i}$, etc.
}

The procedure of building the harmonic balancing polynomial system is summarized in Algorithm 1.

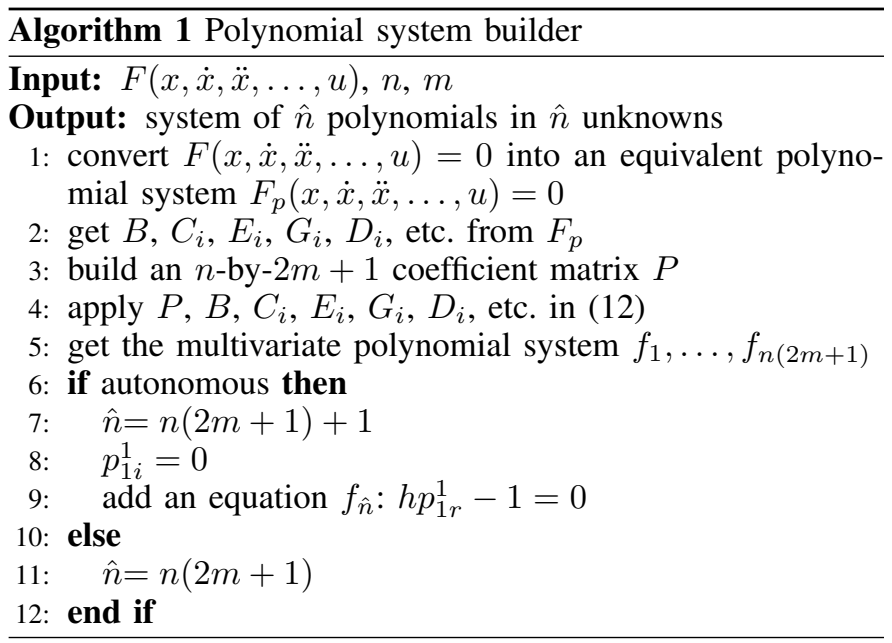

\section{B. Multivariate polynomial root-finder}

We have established in the previous section that the coefficients of a truncated Fourier series of the periodic state solution can be computed as the roots of a multivariate polynomial system. The next step now is to find all the roots of this multivariate polynomial system. The main ingredient of our numerical root finder, which is partly described in [13], [14], is the Macaulay matrix $M(d)$. For a given degree $d \in \mathbb{N}$ and system of $\hat{n}$-variate polynomials $f_{1}, \ldots, f_{\hat{n}}$ of degrees $d_{1}, \ldots, d_{\hat{n}}$, the Macaulay matrix $M(d)$ contains the coefficients of

$$
\left(\begin{array}{c}
f_{1} \\
x_{1} f_{1} \\
\vdots \\
x_{\hat{n}}^{d-d_{1}} f_{1} \\
f_{2} \\
x_{1} f_{2} \\
\vdots \\
x_{\hat{n}}^{d-d_{s}} f_{\hat{n}}
\end{array}\right),
$$

where each polynomial $f_{i}$ is multiplied with all monomials from degree 0 up to $d-d_{i}$ for all $i=1, \ldots, \hat{n}$. Each of these polynomials $f_{1}, \ldots, f_{\hat{n}}$ can be made homogeneous by adding an additional variable $x_{0}$ such that each term has the same degree. The roots of the homogeneous polynomials are then described by the projective coordinates $\left(x_{0}, x_{1}, \ldots, x_{\hat{n}}\right)$. Roots at infinity are roots for which $x_{0}=0$ and affine roots are roots for which $x_{0} \neq 0$. We will denote the homogenization of $f_{1}$ by $f_{1}^{h}$ and $\mathcal{P}_{d}^{\hat{n}}$ will denote the vector space of $\hat{n}$-variate homogeneous polynomials of degree $d$. The row space of $M(d)$ is then the vector space

$$
\mathcal{M}_{d}=\left\{\sum_{i=1}^{\hat{n}} h_{i} f_{i}^{h} \mid h_{i} \in \mathcal{P}_{d-d_{i}}^{\hat{n}}\right\} .
$$

Let $q(d), r(d), c(d)$ respectively denote the number of columns, the rank and the nullity of $M(d)$. Then the ranknullity theorem can be written as

$$
q(d)=r(d)+c(d),
$$


where $q(d)=\left(\begin{array}{c}d+\hat{n} \\ \hat{n}\end{array}\right)=\operatorname{dim} \mathcal{P}_{d}^{\hat{n}}$ and $r(d)=\operatorname{dim} \mathcal{M}_{d}$. By rewriting (15), the following expression for $c(d)$ is found

$$
\begin{aligned}
c(d) & =q(d)-r(d) \\
& =\operatorname{dim} \mathcal{P}_{d}^{n}-\operatorname{dim} \mathcal{M}_{d} \\
& =\operatorname{dim} \mathcal{P}_{d}^{n} / \mathcal{M}_{d}
\end{aligned}
$$

The function in the degree $d$ that describes the dimension of the quotient space $\mathcal{P}_{d}^{\hat{n}} / \mathcal{M}_{d}$ is called the Hilbert Function [17, p. 462]. It can be shown that there is a degree $d^{\star}$ for which the Hilbert Function becomes a polynomial and its degree corresponds with the dimension of the projective solution set [17, p. 464]. This implies that for a polynomial system with a finite number of $s$ projective solutions we have that $c(d)=s\left(\forall d \geq d^{\star}\right)$. It is also possible that the number of affine roots is finite while those at infinity are not finite in number. In this case $c(d)$ will grow polynomially from a certain degree $d^{\star}$. We assume from here on, without loss of generality, that there are a finite number of projective roots and that none of the $s$ roots $z_{1}, \ldots, z_{s}$ have any multiplicities. The canonical null space $K$ of $M(d)$ is then

$$
K=\left(\begin{array}{llll}
\left.\delta_{0}\right|_{z_{1}} & \left.\delta_{0}\right|_{z_{2}} & \ldots & \left.\delta_{0}\right|_{z_{s}}
\end{array}\right)
$$

with

$$
\left.\delta_{0}\right|_{z}=\left(\begin{array}{lllll}
x_{0}^{d} & x_{0}^{d-1} x_{1} & x_{0}^{d-1} x_{2} & \ldots & x_{\hat{n}}^{d}
\end{array}\right)^{T}
$$

in the root $z=\left(x_{0}, x_{1}, \ldots, x_{\hat{n}}\right)$. Note that for an affine root, one can set $x_{0}=1$ in $\left.\delta_{0}\right|_{z}$ and obtain

$$
\left.\delta_{0}\right|_{z}=\left(\begin{array}{lllll}
1 & x_{1} & x_{2} & \ldots & x_{\hat{n}}^{d}
\end{array}\right)^{T} .
$$

This affine canonical null space vector has an $\hat{n}$-variate Vandermonde structure, which implies the following shift property

$$
\left(\begin{array}{lllll}
1 & x_{1} & x_{2} & \ldots & x_{\hat{n}}^{d}
\end{array}\right)^{T} x_{1}=\left(\begin{array}{llllll}
x_{1} & x_{1}^{2} & x_{1} x_{2} & \ldots & x_{1} x_{\hat{n}}^{d}
\end{array}\right)^{T} .
$$

Evidently, this shift property can be written for multiplication with any monomial $x_{i}$. In addition, (16) can be expressed completely in terms of $\left.\delta_{0}\right|_{z}$ by limiting it to the rows of $\left.\delta_{0}\right|_{z}$ corresponding with monomials of degree at most $d-1$. Let $S_{1}$ be the matrix that selects the aforementioned rows and $S_{x_{i}}$ the matrix that selects the corresponding rows after multiplication with $x_{i}$. Then (16) can be written as

$$
\left.S_{1} \delta_{0}\right|_{z} x_{i}=\left.S_{x_{i}} \delta_{0}\right|_{z}
$$

The canonical kernel $K$ can be partitioned into

$$
K=\left(\begin{array}{cc}
s_{a} & s-s_{a} \\
K_{a} & K_{\infty}
\end{array}\right)
$$

where $K_{a}$ contains the $\left.\delta_{0}\right|_{z}$ vectors for the affine roots and $K_{\infty}$ those for the roots at infinity. The shift property can then be written for all $s_{a}$ affine roots as

$$
S_{1} K_{a} D_{x_{i}}=S_{x_{i}} K_{a}
$$

with $D_{x_{i}}$ a $s_{a} \times s_{a}$ diagonal matrix containing the $x_{i}$ component of the corresponding affine roots. The $K_{a}$ matrix is not known however. Instead, a numerical basis $N$ for the null space can be computed using either the singular value decomposition (SVD) or rank-revealing QR [15]. The SVD is the more robust choice for the determination of the rank of $M(d)$ but computationally more expensive. Obviously, $K=N T$ and therefore $K_{a}=N T_{a}$. We now partition $N$ into

$$
\left.\begin{array}{c}
s \\
q-k \\
N_{1} \\
N_{2}
\end{array}\right)
$$

such that $\operatorname{rank}\left(N_{1}\right)=s_{a}$ and compute orthogonal bases $Q_{1}, Q_{2}$ such that $\operatorname{col}\left(Q_{1}\right)=\operatorname{col}\left(N_{1}\right)$ and $\operatorname{col}\left(Q_{2}\right)=\operatorname{null}\left(N_{1}\right)$. This can also be done using either an SVD or rank-revealing $\mathrm{QR}$. Then, the following basis change can be performed $Z=N\left(Q_{1} \quad Q_{2}\right)$. This can be written out as

$$
Z=N Q=\begin{gathered}
s \\
q-k
\end{gathered}\left(\begin{array}{c}
N_{1} \\
N_{2}
\end{array}\right)\left(\begin{array}{cc}
s_{a} & s-s_{a} \\
Q_{1} & Q_{2}
\end{array}\right) .
$$

Since $Q_{2}$ is orthogonal to $N_{1}$ we have that

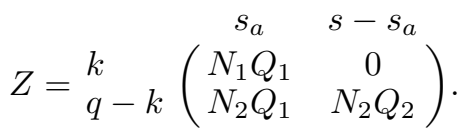

Observe that the upper right block of $Z$ is zero, for this reason this linear basis change is also called a column compression. Also, since $K=N T$ and $Z=N Q$, we can write $K=Z V$ with $V=Q^{T} T$. By applying the same partitioning as (18), $K=Z V$ can be rewritten as

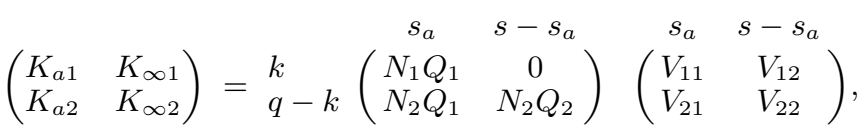

from which we find that $K_{a 1}=N_{1} Q_{1} V_{11}$. Hence, by choosing $S_{1}$ and $S_{x_{i}}$ in (17) such that only rows of $K_{a 1}$ are selected we can write (17) as

$$
S_{1} K_{a 1} D_{x_{i}}=S_{x_{i}} K_{a 1}
$$

Substituting $K_{a 1}$ this becomes

$$
S_{1} N_{1} Q_{1} V_{11} D_{x_{i}}=S_{x_{i}} N_{1} Q_{1} V_{11}
$$

Setting $B \triangleq S_{1} N_{1} Q_{1}$ and $A \triangleq S_{x_{i}} N_{1} Q_{1}$, we obtain

$$
B V_{11} D_{x_{i}}=A V_{11} \text {, }
$$

which is a rectangular eigenvalue problem. This can be solved as

$$
V_{11} D_{x_{i}} V_{11}^{-1}=B^{\dagger} A
$$

where $B^{\dagger}$ is the Moore-Penrose pseudoinverse of $B$. Alternatively, one could choose $S_{1}, S_{x_{i}}$ also such that $B, A$ are square and $B$ of full rank. Then (19) is a generalized eigenvalue problem. Once the eigenvectors $V_{11}$ are computed, they can be used to reconstruct the affine part of the canonical null space $K_{a}$ since $K_{a 1}=N_{1} Q_{1} V_{11}$. All the affine roots can then be read off after scaling the first row of the obtained $K_{a 1}$ such that all its elements are 1 . We summarize the pseudo code for the proposed root-finding method in Algorithm 2.

As described in [13], the number of affine roots $s_{a}$ can be determined from monitoring the linearly independent leading monomials in $\mathcal{M}_{d}$. Indeed, a sufficient condition for the polynomial system to have a finite number of affine roots is that for each variable $x_{i}(i=1, \ldots, \hat{n})$, some pure power $x_{i}^{\alpha}$ is present in the linearly independent monomials of $\mathcal{M}_{d}$. The computational complexity of Algorithm 2 is dominated 
by the computation of a basis for the null space of $M(d)$. For the SVD or rank-revealing QR this complexity is $O\left(p q^{2}\right)$ where $p$ is the number of rows and $q$ the number of columns of $M(d)$. In terms of $\hat{n}$ this complexity is $O\left(\hat{n} d^{3 \hat{n}} /(\hat{n} !)^{3}\right)$. Using a graded monomial ordering for the columns of $M(d)$ results in a particular matrix structure that can be exploited to reduce the complexity to $O\left((\hat{n}-1) d^{3 \hat{n}-3} /(\hat{n}-1 !)^{3}\right)[18]$. This optimization however does not take the quasi-Toeplitz structure nor the sparsity of the Macaulay matrix into account. Indeed, the density of $M(d)$, which is the ratio of the number of nonzero elements with the total number of entries, decreases as $O\left((\hat{n}-1) ! /\left(d^{\hat{n}}\right)\right)$. Further optimizations will hence involve the development of either direct or iterative methods to compute the null space of the sparse and structured Macaulay matrix.

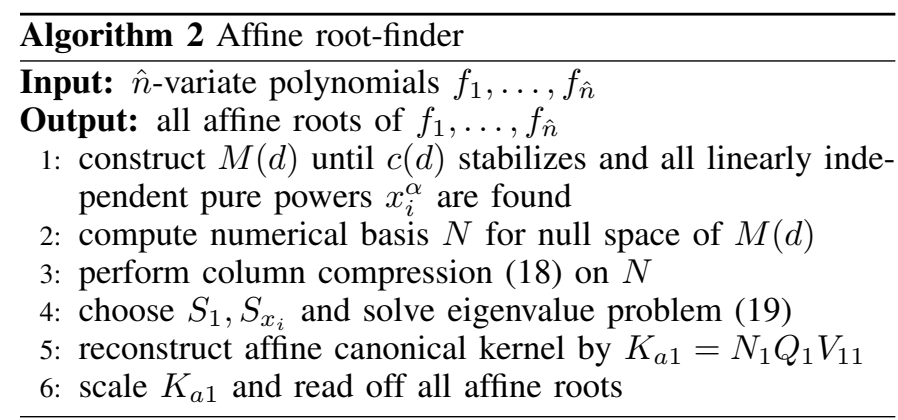

\section{NUMERICAL EXAMPLES}

In this section we illustrate our proposed scheme and compare it with HB by means of several numerical examples. All algorithms were implemented in MATLAB and done on a desktop PC @ $3.0 \mathrm{GHz}$ and 16 GB RAM. In addition, we also compare our computed roots of the polynomial systems with those computed in Maple using Groebner bases.

\section{A. van der Pol oscillator}

Let us consider the following van der Pol-like equation

$$
F_{p}(x)=\ddot{x}-\left(1-3 x^{2}\right) \dot{x}+x=0 .
$$

An electrical circuit implementation for this equation is shown in Figure 1. The tunnel diode has the cubic current-voltage relationship

$$
i=\phi_{t}(v)=\phi\left(v-E_{0}\right)+I \text {, }
$$

with $\phi(v)=\gamma v^{3}-\alpha v$. The equation for the circuit in Figure 1 can then be written as

$$
\dot{V}=\frac{1}{C}(-\phi(V)-W)
$$

with $\dot{W}=V / L$. This can be rewritten to

$$
\ddot{V}-\frac{1}{C}\left(\alpha-3 \gamma V^{2}\right) \dot{V}+\frac{1}{L C} V=0 .
$$

Setting $\alpha=\gamma=1$ and $C=1 F, L=1 H$ results in (20).

We set the order of the Fourier approximation to $m=3$ and use the harmonic balancing scheme from Subsection III-A. Due to the odd symmetry property of (20), all even order

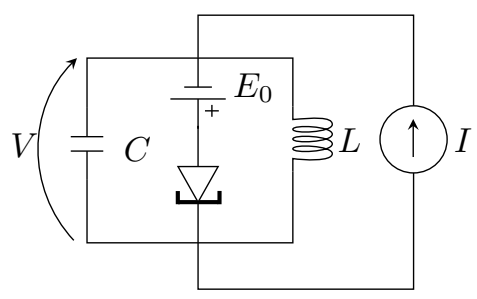

Fig. 1. An electrical circuit with a tunnel diode for the van der Pol oscillator.

Fourier coefficients of $x$ must be 0 (i.e., $p_{0}=p_{ \pm 2}=0$ ). This results in the following polynomial system

$$
\left\{\begin{array}{l}
-\omega^{2}-3 p_{1 r} p_{3 i} \omega+1=0, \\
6 p_{3 r}^{2}+3 p_{1 r} p_{3 r}+6 p_{3 i}^{2}+3 p_{1 r}^{2}-1=0, \\
-9 p_{3 r} \omega^{2}-9 p_{3 i} p_{3 r}^{2} \omega-9 p_{3 i}^{3} \omega-18 p_{1 r}^{2} p_{3 i} \omega \\
+3 p_{3 i} \omega+p_{3 r}=0, \\
9 p_{3 r}^{3} \omega-9 p_{3 i} \omega^{2}+9 p_{3 i}^{2} p_{3 r} \omega+18 p_{1 r}^{2} p_{3 r} \omega \\
-3 p_{3 r} \omega+3 p_{1 r}^{3} \omega+p_{3 i}=0,
\end{array}\right.
$$

of 4 polynomials in 4 unknowns $\left(\omega, p_{1 r}, p_{3 r}, p_{3 i}\right)$. For this system $c(d)$ grows linearly from $d \geq 7$. Only at $d=9$ all pure powers are present in the linearly independent leading monomials. From the linearly independent leading monomials we deduce that the number of affine roots $s_{a}=20$. Note that the linear increase of $c(d)$ together with $s_{a}=20$ implies that solutions set of the roots at infinity is 1-dimensional. $M(9)$ is a $792 \times 715$ matrix with a rank of 607 and a density of $0.7 \%$. This means that $M(9)$ consists of more than $99 \%$ zeros. The computed numerical basis for the null space $N$ is $715 \times 108$. If we choose $S_{1}$ such that it selects all rows of $N$ corresponding with monomials of degree at most 3 , then we have that $\operatorname{rank}\left(N_{1}\right)=20$. Performing the column compression, solving the eigenvalue problem and reconstructing the kernel results in 20 affine roots $\left(\omega, p_{1 r}, p_{3 r}, p_{3 i}\right)$. Only 4 have a real $\omega$, they are all the same solution within a sign change

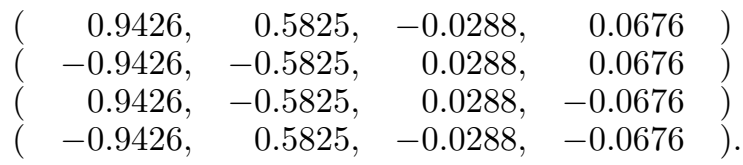

The 2-norm of the vector containing the residuals is of order of magnitude $10^{-13}$. The absolute difference between our computed roots and the roots from the Groebner basis is also of order of magnitude $10^{-13}$.

Next, we apply HB on the oscillator. If the initial guess is carefully chosen to be sufficiently close to the desired solution, e.g. $(1,0.5,0,0)$, then HB converges to the PSS solution in 9 iterations if a tolerance of $10^{-13}$ is used. However, if an unfortunate "bad" initial guess such as $(0.2,1,0,0)$ or $(0.94,0.2,0,0)$ is used, HB will diverge after a few iterations, even when $\omega$ is close to the solution in $(0.94,0.2,0,0)$.

\section{B. Wien bridge oscillator}

The second example is a Wien bridge oscillator adapted from [10]. The circuit and its controlled source model are 


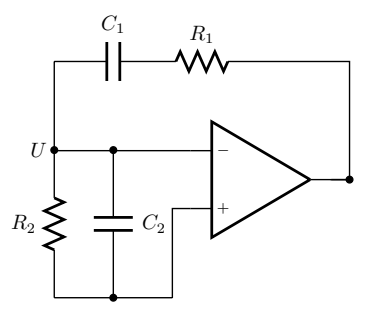

(a)

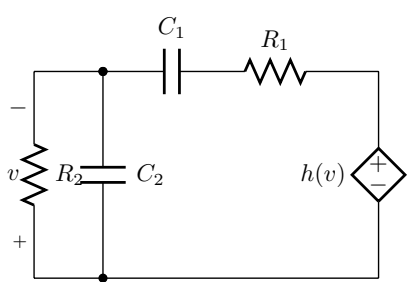

(b)
Fig. 2. (a) A Wien bridge oscillator circuit. (b) Controlled source model of the oscillator.

shown in Figure 2, where $R_{1}=R_{2}=1 \Omega$ and $C_{1}=C_{2}=1 F$. The voltage-controlled voltage source is described by

$$
h(v)=3.234 v-2.195 v^{3}+0.666 v^{5} .
$$

Its univariate DAE can be obtained

$$
F_{p}(v)=\ddot{v}-0.234 \dot{v}+v+6.585 v^{2} \dot{v}-3.334 v^{4} \dot{v}=0 .
$$

Similarly, (22) is odd symmetric, therefore all even order Fourier coefficients should also be 0. Applying the harmonic balancing scheme for $m=3$ results in the polynomial system shown at the top of the next page.

The dimension of the null space $c(d)$ also grows linearly from $d \geq 14$. Only at $d=16$ all pure powers are present in the linearly independent leading monomials. Hence the number of affine roots is again finite and determined to be $s_{a}=80$. $M(16)$ is a $5187 \times 4845$ matrix with a rank of 3999 and a density of $0.2 \%$. The computed numerical basis for the null space $N$ is $4845 \times 846$. Choosing $S_{1}$ such that it selects all rows of $N$ corresponding with monomials of degree at most 5 ensures that $\operatorname{rank}\left(N_{1}\right)=80$. Performing the column compression, solving the eigenvalue problem and reconstructing the kernel results in 80 affine roots $\left(\omega, p_{1 r}, p_{3 r}, p_{3 i}\right)$. Only 8 have

\begin{tabular}{|c|c|c|c|}
\hline 0.7976 & -1.4856 & 0.4119, & -0.0191 \\
\hline 0.7976 & 1.4856 & -0.4119 & -0.0191 \\
\hline-0.7976 & 1.4856, & -0.4119 & 0.0191 \\
\hline 0.7976 & -1.4856 & 0.4119, & 0.0191 \\
\hline 0.9967, & 0.1922, & -0.0005 & 0.0055 \\
\hline-0.9967 , & -0.1922 , & 0.0005 & 0.0055 \\
\hline 0.9967, & -0.1922 , & 0.0005 & -0.0055 \\
\hline-0.9967 & 0.1922, & -0.0005 & -0.0055 \\
\hline
\end{tabular}
a real $\omega$, they are

The 2-norm of the vector containing the residuals is of order of magnitude $10^{-13}$. The absolute difference between our computed roots and the roots from the Groebner basis is of order of magnitude $10^{-12}$. Observe that these results suggest that 2 PSSs exist: one with an angular frequency of 0.7976 and one with an angular frequency of 0.9967. Simulations show that the PSS with $\omega=0.7976$ is unstable, while the other PSS is stable.

Using HB, the stable PSS can be obtained if a "good" initial value, e.g., $(1,0.2,0,0)$, is given. Otherwise, for instance using the initial guess $(1,1,0,0) \mathrm{HB}$ diverges. Furthermore, if $(0.2,1,0,0)$ is chosen, $\mathrm{HB}$ will converge to the unstable solution with $\omega=0.7976$.

\section{Biochemical reaction system}

Then we illustrate a multivariate example with a rational nonlinearity. The system is used to describe a biochemical reaction adapted from [16]. It is an excited system and can be expressed by

$$
\dot{x}+x+\frac{x}{1+10 x}+u=0,
$$

where $u=\cos (2 \pi t)$ is the external input. Using a Taylor expansion is inaccurate to approximate the rational nonlinearity since $x$ is not guaranteed to be within the convergent range of the Taylor expansion. In our scheme, by adding the extra variable $y=x /(1+10 x)$ such that $-x+y+10 x y=0$, we come up with the polynomial DAE system $F_{p}(\dot{x}, x, y, u)=\mathbf{0}$

$$
\left\{\begin{array}{c}
\dot{x}+x+y+u=0 \\
-x+y+10 x y=0
\end{array}\right.
$$

Using $m=2$ in the Fourier approximation results in a polynomial system of 10 equations in 10 unknowns. We have omitted these polynomials due to lack of space. At $d=7$ all pure powers are present in the linearly independent leading monomials. The number of affine roots is determined to be $s_{a}=12 . M(7)$ is a $55055 \times 19448$ matrix with a rank of 19425 and a density of $0.02 \%$. Choosing $S_{1}$ such that it selects all rows of $N$ corresponding with monomials of degree at most 2 ensures that $\operatorname{rank}\left(N_{1}\right)=12$. Performing the column compression, solving the eigenvalue problem and reconstructing the kernel results in 12 affine roots, of which 4 are real. They are given by

$$
\left(\begin{array}{l}
p_{0 r}^{x} \\
p_{0 r}^{y} \\
p_{1 r}^{x} \\
p_{1 r}^{y} \\
p_{2 r}^{x} \\
p_{2 r}^{y} \\
p_{1 i}^{x} \\
p_{1 i}^{y} \\
p_{2 i}^{x} \\
p_{2 i}^{y}
\end{array}\right)=\left\{\left(\begin{array}{r}
0.0551 \\
-0.0551 \\
-0.0251 \\
-0.0368 \\
-0.0028 \\
0.0307 \\
0.0697 \\
0.0878 \\
0.0022 \\
0.0330
\end{array}\right),\left(\begin{array}{r}
-0.1485 \\
0.1485 \\
-0.0049 \\
0.0006 \\
-0.0014 \\
0.0765 \\
0.0789 \\
-0.0479 \\
0.0060 \\
0.0118
\end{array}\right),\left(\begin{array}{r}
-0.2551 \\
0.2551 \\
-0.0251 \\
-0.0368 \\
0.0028 \\
-0.0307 \\
0.0697 \\
0.0878 \\
-0.0022 \\
-0.0330
\end{array}\right),\left(\begin{array}{r}
-0.0515 \\
0.0515 \\
-0.0050 \\
0.0006 \\
0.0014 \\
-0.0765 \\
0.0789 \\
-0.0479 \\
-0.0060 \\
-0.0118
\end{array}\right)\right\} .
$$

It can be shown that only the first two columns of solutions satisfy the hypothesis $y=x /(1+10 x)$. Further transient simulation shows that the first column is the PSS solution to the forced system. The 2-norm of the vector containing the residuals is of order of magnitude $10^{-11}$. The absolute difference between our computed roots and the roots from the Groebner basis is of order of magnitude $10^{-10}$.

Similarly, different initial guesses lead to different results in HB. This is particularly problematic for this example with the occurrence of 4 solutions of which only 1 is desired. For example, $\left(1, \frac{1}{11}, 0, \ldots, 0\right)$ can drive to the solution in the second column while $(0, \ldots, 0)$ results in the right PSS solution.

\section{Colpitts oscillator}

Finally we study an NMOS Colpitts oscillator shown in Figure 3. It is a typical example for autonomous circuits. We use the same normalized state variables as defined in [19]. The normalized state equations of the Colpitts oscillator can 


$$
\left\{\begin{array}{l}
3.334 p_{3 i}^{3} p_{1 r} \omega+10.002 p_{3 i} p_{1 r}^{3} \omega+3.334 p_{3 i} p_{1 r} p_{3 r}^{2} \omega-6.585 p_{3 i} p_{1 r} \omega-1.0 \omega^{2}+1.0=0 \\
13.336 p_{3 i}^{4}+6.668 p_{3 i}^{2} p_{1 r}^{2}+3.334 p_{3 i}^{2} p_{1 r} p_{3 r}+26.672 p_{3 i}^{2} p_{3 r}^{2}-13.17 p_{3 i}^{2}+6.668 p_{1 r}^{4}+16.67 p_{1 r}^{3} p_{3 r}+6.668 p_{1 r}^{2} p_{3 r}^{2}-6.585 p_{1 r}^{2} \\
+3.334 p_{1 r} p_{3 r}^{3}-6.585 p_{1 r} p_{3 r}+13.336 p_{3 r}^{4}-13.17 p_{3 r}^{2}+0.234=0 \\
10.002 p_{3 i}^{5} \omega+40.008 p_{3 i}^{3} p_{1 r}^{2} \omega+20.004 p_{3 i}^{3} p_{3 r}^{2} \omega-19.755 p_{3 i}^{3} \omega+43.342 p_{3 i} p_{1 r}^{4} \omega+6.668 p_{3 i} p_{1 r}^{3} p_{3 r} \omega+40.008 p_{3 i} p_{1 r}^{2} p_{3 r}^{2} \omega \\
-39.51 p_{3 i} p_{1 r}^{2} \omega+10.002 p_{3 i} p_{3 r}^{4} \omega-19.755 p_{3 i} p_{3 r}^{2} \omega+0.702 p_{3 i} \omega-9.0 p_{3 r} \omega^{2}+p_{3 r}=0 \\
10.002 p_{3 i}^{4} p_{3 r} \omega+3.334 p_{3 i}^{2} p_{1 r}^{3} \omega+40.008 p_{3 i}^{2} p_{1 r}^{2} p_{3 r} \omega+20.004 p_{3 i}^{2} p_{3 r}^{3} \omega-19.755 p_{3 i}^{2} p_{3 r} \omega+9.0 p_{3 i} \omega^{2}-1.0 p_{3 i}+43.342 p_{1 r}^{4} p_{3 r} \omega \\
+10.002 p_{1 r}^{5} \omega+10.002 p_{1 r}^{3} p_{3 r}^{2} \omega-6.585 p_{1 r}^{3} \omega+40.008 p_{1 r}^{2} p_{3 r}^{3} \omega-39.51 p_{1 r}^{2} p_{3 r} \omega+10.002 p_{3 r}^{5} \omega-19.755 p_{3 r}^{3} \omega+0.702 p_{3 r} \omega=0 .
\end{array}\right.
$$

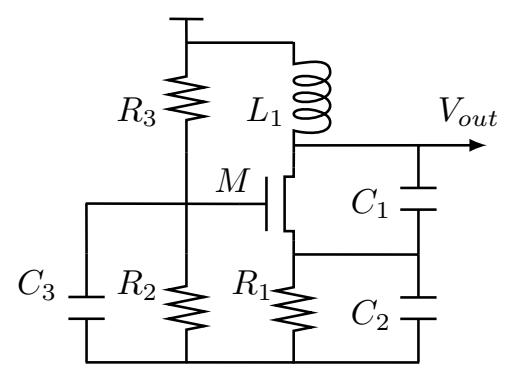

Fig. 3. An NMOS Colpitts oscillator.

be written in the form

$$
\left\{\begin{array}{l}
\dot{x}_{1}=\frac{3}{2}\left(x_{2}+x_{3}\right)-\frac{3}{4} x_{2}^{2} \\
\dot{x}_{2}=\frac{3}{2} x_{3} \\
\dot{x}_{3}=-\frac{1}{3}\left(x_{1}+x_{2}\right)-\frac{1}{2} x_{3} .
\end{array}\right.
$$

To verify the existence of the periodic solution, we apply our scheme for $m=1$ and this results in a polynomial system of 10 equations. Here, we also omitted these equations due to the lack of space. It is possible to reduce this system. Indeed, from the polynomial system it is immediately clear that $p_{0 r}^{3}=0$ and this also allows us to substitute $p_{0 r}^{2}$ by $-p_{0 r}^{1}$, effectively reducing the total number of polynomials and unknowns to 8 . The dimension of the null space $c(d)$ grows cubicly from $d \geq 4$, which implies that the solution set at infinity is 3dimensional. All 6 affine solutions are found at $d=8$, at which the Macaulay matrix is $24024 \times 12870$ with a density of $0.02 \%$. Each of the 6 affine roots is real. After removing the solutions within sign changes, only the following 2 solutions remain

$$
\left(\begin{array}{c}
\omega \\
h \\
p_{0 r}^{1} \\
p_{0 r}^{2} \\
p_{0 r}^{3} \\
p_{1 r}^{1} \\
p_{1 r}^{2} \\
p_{1 r}^{3} \\
p_{1 i}^{2} \\
p_{1 i}^{3}
\end{array}\right)=\left\{\left(\begin{array}{r}
1.0000 \\
1.3416 \\
-0.3333 \\
0.3333 \\
0.0000 \\
0.7454 \\
0.3727 \\
-0.2485 \\
0.3727 \\
0.2485
\end{array}\right),\left(\begin{array}{r}
0.0000 \\
1.4142 \\
-1.0000 \\
1.0000 \\
0.0000 \\
0.7071 \\
-0.7071 \\
0.0000 \\
0.0000 \\
0.0000
\end{array}\right)\right\}
$$

The 2-norm of residuals is of order of magnitude $10^{-14}$ and the absolute difference between our computed roots and the roots from the Groebner basis is also of order of magnitude $10^{-14}$. The result suggests that there are 2 possible PSS for the Colpitts oscillator. The first column of the solution is the desired PSS, with $\omega=1$ satisfying the normalization condition. The second column indicates the unstable constant equilibrium point of the Colpitts oscillator, which can be easily destroyed by a small perturbation.

Not surprisingly, different initial conditions lead to different solutions in $\mathrm{HB}$. For example, $(1,1,0,0,0,1,-1,0$, $0,0)$ leads to the unstable equilibrium point while $(1,1,1,0$, $0,1,0,0,0,0)$ blows up the iteration. Finally $\mathrm{HB}$ converges to the right PSS solution starting from the initial guess $(1,1,0,0,0,1,1,0,0,0)$.

\section{REMARKS}

Several important remarks are in order:

1) Although our balancing equation (12) resembles the harmonic balance (HB) method [3], [4], [7], it is important to observe that $\mathrm{HB}$ deals with nonlinear functions by evaluating in the time domain, followed by fast Fourier transforms (FFT) in each iteration of Newton's method. In the proposed method, the nonlinearity is embedded in the polynomial balancing formulation and no domain transforms are required.

2) The Groebner basis with respect to the degree negative lex monomial ordering for the Wien bridge example has integer coefficients with up to 150 digits and requires multiprecision integer arithmetic. The proposed method works in double precision and is able to compute results with similar accuracy.

3) In subsection II-A, it should be noticed that the size of the state space in (1) will be enlarged after the polynomialization process, i.e., $n>\tilde{n}$ in (1) and (2). The number of extra state variables depends on the number of nonlinearities of each electrical component (including recursive nonlinearities). In practical circuit modeling the number of nonlinearities of each component is always limited and countable. Therefore the complexity of the algorithm will not be increased due to the conversion.

4) If the obtained accuracy of our proposed method is not sufficient, then only a few Newton iterations, using the obtained solutions as initial guess, are sufficient to further improve the accuracy up to the machine precision.

5) Future work will focus on solving higher order Fourier approximations. This will result in larger polynomial systems, which will be the main bottleneck for the proposed method. Exploiting the structure and nonzero pattern of the Macaulay matrix is then mandatory. 


\section{CONCLUSION}

In this paper, a novel method for solving the PSS problem of nonlinear oscillators has been proposed. Prior nonlinear autonomous system solvers suffer from either convergence issues or intrinsic coefficient problems, which are circumvented by the proposed method via a Macaulay matrix based polynomial solver. Moreover, exact polynomial DAE systems are constructed from general nonlinear problems to guarantee the strong nonlinearities of the original systems. Several examples confirm the accuracy and feasibility of the proposed method.

\section{REFERENCES}

[1] K. Mayaram, D. C. Lee, S. Moinian, D. A. Rich, and J. Roychowdhury, "Computer-aided circuit analysis tools for RFIC simulation: algorithms, features, and limitations," IEEE Trans. Circuits Syst. II, vol. 47, no. 4, pp. 274-286, Apr. 2000.

[2] J. Aprille, T.J. and T. Trick, "Steady-state analysis of nonlinear circuits with periodic inputs," Proceedings of the IEEE, vol. 60, no. 1, pp. 108 - 114, jan. 1972.

[3] O. Nastov, R. Telichevesky, K. Kundert, and J. White, "Fundamentals of fast simulation algorithms for RF circuits," Proc. IEEE, vol. 95, no. 3, pp. 600-621, Mar. 2007.

[4] K. Kundert and A. Sangiovanni-Vincentelli, "Simulation of nonlinear circuits in the frequency domain," Computer-Aided Design of Integrated Circuits and Systems, IEEE Transactions on, vol. 5, no. 4, pp. 521 535, october 1986.

[5] R. Telichevesky, K. Kundert, and J. White, "Efficient steady-state analysis based on matrix-free Krylov-subspace methods," in Design Automation, 1995. DAC '95. 32nd Conference on, 1995, pp. 480-484.

[6] M. Nakhla and J. Vlach, "A piecewise harmonic balance technique for determination of periodic response of nonlinear systems," Circuits and Systems, IEEE Transactions on, vol. 23, no. 2, pp. 85-91, 1976.

[7] K. S. Kundert, J. K. White, and A. Sangiovanni-Vincentelli, Steadystate methods for simulating analog and microwave circuits. Kluwer Academic Publishers Boston, 1990.
[8] K. D. Boianapally, T. Mei, and J. Roychowdhury, "A multi-harmonic probe technique for computing oscillator steady states," in ComputerAided Design, 2005. ICCAD-2005. IEEE/ACM International Conference on. IEEE, 2005, pp. 610-613.

[9] J. Parkhurst and L. Ogborn, "Determining the steady-state output of nonlinear oscillatory circuits using multiple shooting," Computer-Aided Design of Integrated Circuits and Systems, IEEE Transactions on, vol. 14, no. 7, pp. 882-889, 1995.

[10] L. Chua and Y.-S. Tang, "Nonlinear oscillation via volterra series," Circuits and Systems, IEEE Transactions on, vol. 29, no. 3, pp. 150$168,1982$.

[11] H. Liu and N. Wong, "Autonomous volterra algorithm for steady-state analysis of nonlinear circuits," Computer-Aided Design of Integrated Circuits and Systems, IEEE Transactions on, vol. 32, no. 6, pp. 858868, 2013.

[12] K. Forsman and T. Glad, "Constructive algebraic geometry in nonlinear control," in IN PROC. 29TH CDC. IEEE, 1990, pp. 2825-2827.

[13] K. Batselier, P. Dreesen, and B. De Moor, "Prediction error method identification is an eigenvalue problem," ser. Proc 16th IFAC Symposium on System Identification (SYSID 2012), 2012, pp. 221-226.

[14] P. Dreesen, K. Batselier, and B. De Moor, "Back to the roots: Polynomial system solving, linear algebra, systems theory," ser. Proc 16th IFAC Symposium on System Identification (SYSID 2012), 2012, pp. $1203-1208$.

[15] G. H. Golub and C. F. Van Loan, Matrix Computations, 3rd ed. The Johns Hopkins University Press, Oct. 1996.

[16] C. Gu, "QLMOR: A projection-based nonlinear model order reduction approach using quadratic-linear representation of nonlinear systems," Computer-Aided Design of Integrated Circuits and Systems, IEEE Transactions on, vol. 30, no. 9, pp. 1307-1320, 2011.

[17] D. A. Cox, J. B. Little, and D. O'Shea, Ideals, Varieties and Algorithms, 3rd ed. Springer-Verlag, 2007.

[18] K. Batselier, P. Dreesen, and B. De Moor, "A fast iterative orthogonalization scheme for the Macaulay matrix," vol. 267, pp. 20-32, 2014, Journal of Computational and Applied Mathematics.

[19] G. Maggio, O. De Feo, and M. Kennedy, "Nonlinear analysis of the Colpitts oscillator and applications to design," Circuits and Systems I: Fundamental Theory and Applications, IEEE Transactions on, vol. 46, no. 9, pp. 1118-1130, Sep 1999. 